\title{
Effects Analysis on the Mechanical Properties of Nanocrystalline Materials
}

\section{Yue-Wu Liu}

Division of Engineering Sciences, Institute of Mechanics, Chinese Academy of Sciences,Beijing 100080, China, Email: lywu@imech.ac.cn

\section{Rong Zhou}

State Key Laboratory of Nonlinear Mechanics, Institute of Mechanics, Chinese Academy of Sciences, Beijing 100080, China, Email: zhour@imech.ac.cn

\section{Shou-Qin Lü}

National Microgravity Laboratory, Institute of Mechanics, Chinese Academy of Sciences, Beijing 100080, China, Email: lshouqin@x263.net

\begin{abstract}
Nanocrystalline materials are characterized by a typical grain size from 1 to $100 \mathrm{~nm}$. In order to study the nanocrystalline properties of nanocrystalline materials, we chose nanocrystalline coppers as the research object. The uniaxial tensile deformation of computer produced nanocrystalline coppers is simulated by using molecular dynamics with Finnis-Sinclair potential. The mean grain size of simulated nanocrystalline coppers is varied within the 5.38 to $1.79 \mathrm{~nm}$ range. The strength, Young's modulus and stress-strain are strongly depended on the grain size and nanocrystalline structure. The simulated nanocrystalline coppers show a reverse Hall-Petch effect.
\end{abstract}

Keywords: Nanocrystalline materials, mechanical properties, molecular dynamics

\section{Introduction}

Nanocrystalline materials are characterized by a typical grain size from 1 to $100 \mathrm{~nm}$. Mechanical properties of nanocrystalline materials display an increasing interest because they are significantly different from those of the coarse-grained counterpart. Dramatic changes in mechanical properties such as hardness, strength and ductility, occur when the grain size is progressively reduced to the nanometer scales ${ }^{[1-3]}$. It has been pointed out that size effects may be responsible for specific changes in the mechanisms of deformation ${ }^{[7]}$. The plastic behavior of metals, which in the bulk situation strong depends on dislocation activity, start to differ substantially entering the nanometer regime because of reduced availability of mobile dislocations in the small nanograins. Conventional physical models for crystal plasticity have been revised to include size effects due to the presence of a large density of grain boundaries. Reported experiments on elastic and plastic behavior concentrate mainly on determination of Young's modulus, hardness measurements and creep tests ${ }^{[5 \text { - }}$ 8].

New computational approaches augmenting the time-consuming search-and-test methods traditionally used for the development of new materials. The atomistic calculations provide a useful tool to analyze structural, mechanical and thermodynamic properties of nanocrystalline materials and, eventually to design materials of assigned characteristics. Molecular dynamics (MD) 
approach, as one of most important methods of atomistic simulation, provides the phase-space trajectories of particles through the solution of Newton's equation, thereby shedding light on that how atomic level processes lead to macroscopic phenomena, so it can help understanding the relationship between structure and overall properties. MD simulation may display trajectories of atomic motion, and may be used to carry a variety of observations similar to those in experiment, especially those details, related with atoms, can be easily acquired through MD simulation but can not in experiment. MD simulation, not limited by synthesis, thermal history and testing technique of sample, may be used to elucidate the experimental results in theory, and make up for the shortage of experiment.

In this paper we present some molecular dynamics simulation results to analyze the effects on the mechanical properties of the nanocrystalline materials. The main mechanical properties are the strength, elastic modulus, Hall-patch relationship. The simulation results indicate that the gain size and the structure of grain boundary are the main factors which affect the mechanical properties of the materials.

\section{Simulation Method}

To simulate the deformation of nanocrystalline metals with computer, we construct nanocrystalline samples with structures similar to those observed experimentally. Five samples containing approximately 55,300 atoms each, with $8,27,64$, 125 , and 216 grains $\left(\mathrm{Cu} \_5.38, \mathrm{Cu} 3.59\right.$, $\mathrm{Cu} 2.69, \mathrm{Cu} 2.15$, and $\mathrm{Cu}$ 1.79), which represent average grain sizes ranging from 5.38 to $1.79 \mathrm{~nm}$, are constructed by filling the simulation cell volume of a $8.67-\mathrm{nm}$ cube. The grains are produced by a Voronoi polyhedron construction ${ }^{[1]}$ in which a set of grain centers and crystallographic orientation of each grain are chosen at random. In order to eliminate unfavourable configurations in grain boundaries, the samples are relaxed by running a 50-ps at $300 \mathrm{~K}$ and $\mathrm{latm}$, using molecular dynamics simulation with a FinnisSinclair potential ${ }^{[12,13]}$ in the Parrinello-Rahman approach $^{[14]}$, and periodic boundary conditions that replicate the cube infinitely in all three spatial directions. The atomic trajectories were followed by integrating the Newton's equation of motion for each atoms with the Frog-Leap algorithm $\left.{ }^{[15}\right]$. The time unit in simulation is $\tau_{0}=10^{-14} \mathrm{~s}$, the energy unit $1.0 \mathrm{eV}$, and the length unit $a_{0}=3.615 \mathrm{~A}$.

\section{The Strength}

The experiment results have showed that the strength strongly depend on the gain sizes[13]. The results is showed in Fig.1.Our simulation results are quit greet with this conclusion.

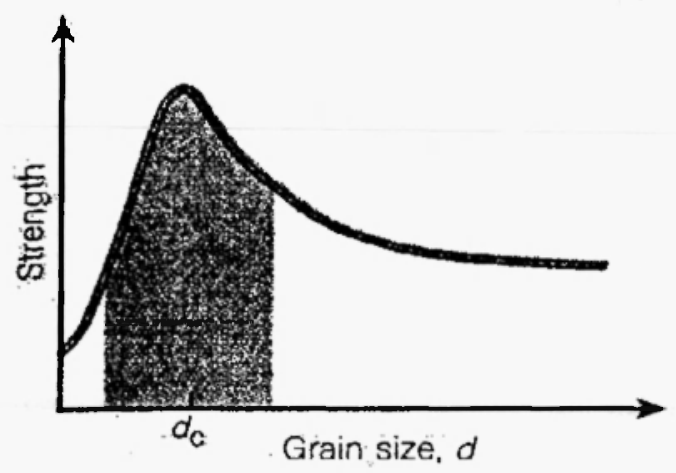

Fig.1 The relationship between the strengths and the gain sizes.

\section{Young's Modulus}

There are a number of literatures in which the measured value of elastic modulus is lower than those of their coarse-grain counterparts and a number of theories were developed to explain the modulus decrement, such as porosity, inaccurate measurement and low density samples $^{[2,8]}$. Fig.2 shows simulated elastic modulus versus inverse grain size. The figure shows the strong dependence of elastic modulus on grain size. As the grain size is reduced from $5.38 \mathrm{~nm}$ to $1.79 \mathrm{~nm}$, the Young's modulus has decreased $24 \%$ (from $114.5 \mathrm{GPa}$ to $80.7 \mathrm{GPa}$ ). The simulated elastic modulus of nanocrystalline $\mathrm{Cu}$ is smaller than that of polycrystalline $\mathrm{Cu}$. The reason which results in the elastic modulus decrement may be the lower elastic moduli of grain boundaries. Due to the free volume of grain boundaries, the average interatomic spacings in boundary regions are increased relative to perfect lattice. A further 
structure analysis also shows under-12coordinated atoms in grain boundaries have increased distinctly with grain size decreasing. If the interatomic potential in grain boundaries is assumed to be the same as in the perfect lattice, the elastic modulus of ananocrystalline materials are expected to be reduced in comparison to crystalline state due to the reduced elastic moduli of grain boundaries. As the grain size is small $(<5 \mathrm{~nm})$, the volume fraction of grain boundaries becomes large $(>30 \%)$ hence the influence of grain boundary on the elastic modulus is quite remarkable.

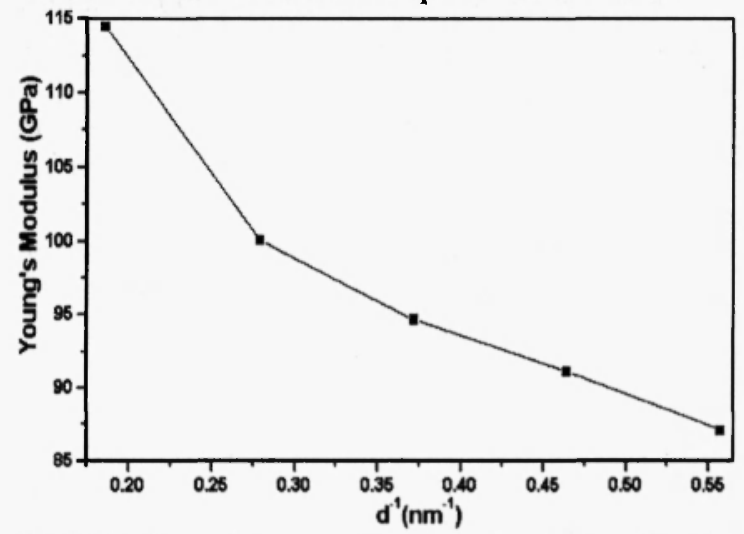

Fig.2 Elastic modulus as a function of grain size. The figure shows the strong dependence of elastic modulus on grain size, i.e. the decreasing of elastic modulus with reduced grain size.

\section{Stress-strain Responses}

The simulated true stress-strain curves of nanocrystalline copper are showed Fig.3. A significant strength increase is clearly observed when the grain size increases from $1.79 \mathrm{~nm}$ to $5.38 \mathrm{~nm}$. Compared with experimental results of coarse-grained counterpart or nanocrystalline $\mathrm{Cu}$, the level of stress of simulated $\mathrm{Cu}$ is distinctly higher, which may come from many respects, such as pores (and larger flaws), lattice or surface defects and impurities of nanocrystalline materials in experiment that depend on the manner in which these materials are synthesized and processed, reducing the strength of materials. The flow stress of nanocrystalline samples, which is defined as the maximal stress of the stress-strain curves, has increased as the average grain size becomes larger. The result is not consistent with that of the coarse-grained counterpart materials mechanical response dominated by a high preexisting dislocation structure.

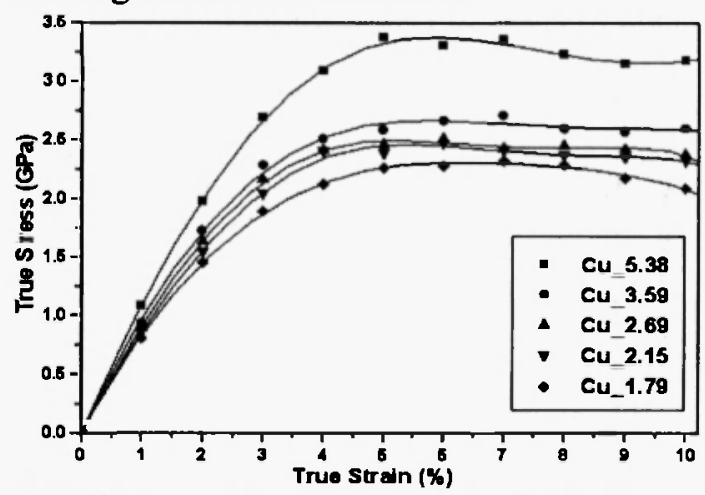

Fig. 3 The true stress versus the true strain for five samples. The curves are polynomial fitting of simulated results of each samples.

\section{Hall-Petch Relationship}

The strength of a polycrystalline materials can be enhanced by decreasing the grain size. The phenomenological relation between the yield stress $\sigma \mathrm{y}$ and the mean grain size $\mathrm{d}$ can be expressed by the well-known Hall-Petch equation $^{[14]}$ :

$$
\sigma_{y}=\sigma_{0}+k d^{-1 / 2}
$$

where $\sigma_{0}$ (friction stress) and $\mathrm{k}$ (H-P coefficient) are material constants. The equation for hardness is analogous. The Hall-Petch equation predicts that nanocrystalline materials would display great strength and ultrahardness. In this article we find that nanocrystalline copper possess a much higher strength than its coarsegrained counterpart but the results show a reverse Hall-Petch relationship, i.e. a decrease in strength with decreasing grain size.

According to Fig.3, the yield stress plotted as a function of $\mathrm{d}^{-1 / 2}$ is illustrated in Fig.4. A least-squares linear fit in according to eq.(1) gives the follows estimate for $\mathrm{k}$ and $\mathrm{o}_{0}$ :

$$
\begin{aligned}
& k=-(1.645 \pm 0.0878) \mathrm{GPa} \cdot \mathrm{nm}^{1 / 2} \\
& \sigma_{0}=(2.498 \pm 0.0535) \mathrm{GPa}
\end{aligned}
$$

The negative H-P coefficient $\mathrm{k}$ shows a softening with grain size under $6 \mathrm{~nm}$, and the absolute value of $k$ is over three times 
experimental results of ne $\mathrm{Cu}$ $\left(k=470 G P a \cdot \mathrm{nm}^{1 / 2}\right)$ with $12 \sim 85 \mathrm{~nm}^{[15]}$. The reverse Hall-Petch effect has already been investigated in experimental researches ${ }^{[16]}$, but the value of $k$ has not been offered.

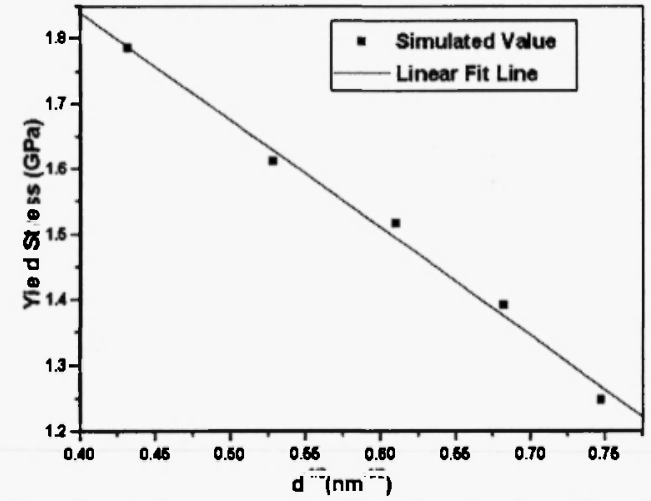

Fig. 4 The yield stress as a function of grain size. The yield stress defined as the stress where the strain departs $0.2 \%$ from linearity, decreases with decreasing of grain size, resulting in reverse Hall-Petch effect.

\section{Conclusion}

The simulation results indicate that the gain size and the structure of grain boundary are the main factors which affect the mechanical properties of the materials. The strength, Young's modulus and stress-strain are strongly dependent on the grain size and nanocrystalline structure. The simulated nanocrystalline coppers show a reverse Hall-Petch effect.

\section{Acknowledgments}

This work is supported by the National Natural Science Foundation (10172088).

\section{References}

[1] Siegel R W. Mechanical properties of nanophase materials. Materials Science Forum, 1997, 235238: 851-860

[2] Sanders P G, Youngdahl C J and Weertman J R. The strgngth of Nanocrystalline Metals with and without flaws. Materials Science and Engineering A, 1997, 234-236: 77-82

[3] Koch C C and Malow T R. The ductility problem in nanocrystalline materials. Materials Science Forum, 1999, 12-314: 565-574

[4] Gleiter H. Nanostructured materials: state of the art and perspectives. Nanostructured Materials, 1995, 16: 3-14

[5] Lu L, Sui M L, Lu K. Superplastic extensibility of nanocrystalline copper at room temperature. Science, 2000, 287: 1463-1466

[6] Qin X Y, Zhang X R, Cheng G S, and Zhang L D. The elastic properties of nanostructured $\mathrm{Ag}$ measured by laser ultrasonic technique. Nanostructured Materials, 1998, 10(4): 661-672

[7] Yang M C, Ye F, Sun X C, Sun X K, Wei W D. Study on microhardness of bulk nanocrystalline copper. Nanostructured Materials, 1997, 9: 481484

[8] Sanders P G, Rittner M, Kiedaisch E, Weertman J $\mathrm{R}$, Kung $\mathrm{H}$ and $\mathrm{Lu} \mathrm{Y} \mathrm{C}$. Creep of nanocrystalline $\mathrm{Cu}, \mathrm{Pd}$, and $\mathrm{Al}-\mathrm{Zr}$. Nanostructured Materials, 1997, 9: 433-440

[11] Finney J L. Fine structure in randomly packed, dense clusters of hard spheres. Materials Science and Engineering, 1976, 23: 199-205

[12] Finnis M W, Sinclair J E. A simple empirical nbody potential for transition-metals. Philo. Mag. A, 1984, 50(1): 45-55

[13] Sidney Yip.The strongest size. Nature, 1998,391(5):532-533

[14] Hertzberg R W. Deformation and fracture mechanics of engineering materials. New York: John Wiley \& Sons, 1976. 103-156

[15] Weertman J R. Hall-Petch strengthening in nanocrystalline metals. Materials Science and Engineering A, 1993, 166: 161-167

[16] Song H W, Guo S R and Hu Z Q. A Coherent polycrystal model for the inverse Hall-Petch relation in nanocrystalline materials. Nanostructured Materials, 1997, 11(2): 203-210

[17] Wen Yuhua, Zhou Fuxin, Liu Yuewu. Molecular dynamics simulation of microstructure of nanocrystalline copper. Chinese Physics Letter, 2001,18(3): $411-413$

[18] Wen Yuhua, Zhou Fuxin, Liu Yuewu. The influence of grain size and temperature on the mechanical deformation of nanocrystalline materials: molecular dynamics simulation. Chinese Physics. 2001,10(5): 407-4 12

[19] Wen Yuhua, Zhou Fuxin, Liu Yuewu,Zhou Chengen. Molecular dynamics simulation of the uniaxial tesile deformation of nanocrystalline copper. Acta Mechanica Sinica, 2002,34(1): 2936. 